\title{
Climatologia de Sistemas Convectivos de Mesoescala ocorridos sobre a América do Sul no período de 2005 a 2006
}

\author{
Climatology of mesoscale convective systems occurring over South America in the \\ period $2005-2006$
}

\author{
Vanessa Ferreira $^{1}$, Vagner Anabor ${ }^{1}$ \\ ${ }^{1}$ Grupo de Modelagem Atmosférica de Santa Maria - Universidade Federal de Santa Maria,
}

\begin{abstract}
Resumo
Uma curta climatologia é apresentada com o objetivo de documentar a frequência e os padrões morfológicos dos Sistemas Convectivos de Mesoescala ocorridos no Sul da América do Sul entre 2005-2006. Usando-se dados do satélite GOES-13 e um rastreador automático de tempestades, 33 eventos de SCMs foram identificados e classificados segundo sua excentricidade, 60\% foram classificados como SCMs alongados e 40\% como SCMs circulares/ ou Complexos Convectivos de Mesoescala (CCMs). A análise de dados mostrou mais alta frequência dos SCMs relativamente aos CCMs, maior área, bem como tempo de vida mais longo. Em média o tempo de vida foi de 44 h para os SCMs, e 26h para os CCMs. Por isso, os SCMs alongados geram impactos socioeconômicos mais importantes do que os CCMs, sendo mais significantes para o ciclo hidrológico na região Sul da América do Sul.
\end{abstract}

Palavras-chave: Sistemas convectivos de mesoescala. Complexos convectivos de mesoescala.

\begin{abstract}
In order to document the frequency and morfological features of Mesoscale Convective Systems (MCSs) over Southern South América between 2005-2006, a short climatology is presented. Using GOES-13 Satellite data and and automatic strom track classification procedure, 33 MCSs events were identified and cataloged following its excentricity. $60 \%$ of cases were classified as MCSs enlongated and $40 \%$ as MCSs circularl or Mesoscale Convective Complexes (MCCs). The data analysis shows the MCSs higher frequency relatively to MCCs, cover larger areas, and also longest life time. In average time life of $44 \mathrm{~h}$ for SCMs, and $26 \mathrm{~h}$ for CCMs. Therefore, SCMs enlongated create a higher socio-economical impacts than MCCs, being more significant for Souther South America hidrological cycle.
\end{abstract}




\section{Introdução}

Os SCMs frequentemente estão associados a condições de tempo severo, pois podem produzir fortes rajadas de vento e precipitações intensas, causando impactos sociais e econômicos devido ao seu grande potencial de destruição.

Maddox (1980, 1983) reportou um tipo circular de SCM, denominado Complexo Convectivo de Mesoescala (CCM), que são sistemas caracterizados por sua grande extensão em área, longo tempo de vida, e um escudo de nuvens quase circular em imagens de satélite no canal do infravermelho termal.

\section{Quadro 01: Características físicas dos CCMs}

\begin{tabular}{|l|l|}
\hline Tamanho & $\begin{array}{l}\text { a) } \mathrm{O} \text { escudo de nuvens com } \\
\text { temperaturas } \leq-32^{\circ} \mathrm{C} \text { deve ter uma } \\
\text { área } \geq 100000 \mathrm{~km}^{2}\end{array}$ \\
\cline { 2 - 2 } & $\begin{array}{l}\text { b) A região fria no interior do } \\
\text { sistema com temperatura } \leq-52^{\circ} \mathrm{C} \\
\text { deve ter uma área } \geq 50000 \mathrm{~km}^{2} .\end{array}$ \\
\hline Iniciação & $\begin{array}{l}\text { Os critérios definidos em a e b } \\
\text { devem ser satisfeitos. }\end{array}$ \\
\hline Extensão & $\begin{array}{l}\text { A extensão máxima do CCM ocorre } \\
\text { quando o escudo de nuvens com } \\
\text { temperatura } \leq-32^{\circ} \mathrm{C} \text { atinge seu } \\
\text { tamanho máximo. }\end{array}$ \\
\hline Forma & $\begin{array}{l}\text { Excentricidade }>0.7 \text { no momento } \\
\text { de máxima extensão. }\end{array}$ \\
\hline Dissipação & $\begin{array}{l}\text { Os critérios de tamanho a e b não } \\
\text { são mais satisfeitos. }\end{array}$ \\
\hline
\end{tabular}

Adaptado de Maddox (1980).

Anderson e Arrit (1998) descrevem sistemas convectivos que possuem os mesmos critérios de tamanho e formação dos CCMs, porém possuem um escudo de nuvens com forma mais alongada e duração maior que 12 horas, denominados Sistemas Convectivos Persistentes e Alongados (PECS). Anabor et. al (2008) estuda SCMs alongados com propagação e evolução contra o escoamento e com duração maior de 18 horas.

Na América da Sul a incidência de SCMs é maior durante a estação quente, e a ocorrência destes sistemas é documentada por Anabor (2004,2008), Velasco e Fritsch (1987), indicando que em média eles possuem características morfológicas similares aos da América do Norte. Porém, na América do Sul os SCMs tendem a se desenvolver um pouco mais ao anoitecer, duram mais tempo e são em média maiores que os sistemas na América do Norte (Velasco e Fritsch, 1987). E por Durkee e Mote (2009), mostrando que os sistemas sobre a Amé- rica do Sul possuem grande potencial para contribuir significativamente na precipitação total em toda a região.

Portanto, este estudo apresenta uma climatologia de SCMs que ocorreram sobre a América do Sul no período de 2005 a 2006, tendo como objetivo documentar a frequência, as principais características morfológicas e a variação temporal destes sistemas.

\section{Materiais e métodos}

Os eventos de SCMs foram selecionados através de um rastreador automático de tempestades desenvolvido denominado PyStormTracker, que se utiliza de imagens de satélite GOES e de uma biblioteca de visão computacional chamada OpenCV, que é utilizada em computação para rastreio geométrico de objetos em imagens, jogos e aplicações de realidade aumentada.Para esta seleção o rastreador utiliza os limiares de temperatura menor que $-55^{\circ} \mathrm{C}$ e área convectiva maior de $100000 \mathrm{~km}^{2}$.

Existem outros sistemas de classificação e rastreio de SCMs, como a Técnica de Máxima Correlação Espacial e Rastreio (MASCOTTE) de Carvalho e Jones (2001), o sistema de Previsão a Curto Prazo e Evolução de Sistemas Convectivos (FORTRACC) da Divisão de Satélites Ambientais/INPE e um processamento semi-automático desenvolvido por Anabor (2004, 2008). A vantagem do PyStormTracker, é que ele se utiliza de técnicas de visão computacional da biblioteca OpenCV, que possui funções para identificação de forma, rastreio de objetos em imagens estáticas ou vídeos, permitindo o cálculo de trajetórias, áreas, e que pode ser facilmente adaptada, sendo por isso aplicável ao rastreio de tempestades, e portanto torna o processo de classificação e rastreio dos SCMs mais eficaz.

Para o cálculo da temperatura, inicialmente o rastreador PyStormTracker converte os níveis digitais da imagem em valores de radiância e depois em temperatura de brilho, e então identifica os contornos das nuvens com temperatura menor que $-55^{\circ} \mathrm{C}$.

Propriedades da tempestade como comprimento dos eixos e excentricidade, posição do centro de massa na imagem, são calculadas através de funções já existentes na biblioteca de visão computacional OpenCV. O sistema também registra o histórico de todas as nuvens que confluíram ao longo do tempo e formaram o SCM. Após o processamento, as informações obtidas a partir da imagem são armazenadas em um banco de dados relacional e as consultas destas informações são feitas através de linguagem SQL, posteriormente será feita uma interface para esta consulta.

Portanto propriedades da tempestade, como área, comprimento dos eixos, excentricidade, centro de massa e posição ao longo do tempo de vida do SCM, foram calculadas pelo PyStormTracker e salvas em um banco de dados. A partir destas informações foram feitas as análises estatísticas dos dados para verificar-se a frequência de 
ocorrência dos SCMs e suas características morfológicas. Separou-se os eventos entre SCMs alongados e CCMs, e para cada calculou-se a frequência mensal, a duração média em horas e o coeficiente correlação entre área máxima e duração.

\section{Resultados e discussão}

Identificaram-se 33 eventos de SCMs no período de 2005 e 2006, e destes, através do parâmetro de excentricidade, 20 eventos, representando $60 \%$ do total de eventos, classificam-se como SCMs alongados e 13 como CCMs (excentricidade > 0.7), representando $40 \%$ do total de eventos.

A análise mostra que 75\% dos SCMs 85\% dos CCMs ocorrem na estação quente, entre os meses de outubro e março (Figuras 03 e 04), e são sistemas de longa duração, onde os SCMs tem duração média de 44 horas e os CCMs de 26 horas (Figuras 07 e 08). Os resultados demonstram que a maioria dos sistemas atingem áreas máximas entre 1×105 e 5 x 105 km² (Figura 05) e que os SCMs alongados atingem áreas máximas maiores e tem duração maior que os CCMs. Observa-se que os CCMs tem curtose menor que os SCMs (Figura 06), indicando que os SCMs ocorrem em ambientes sinóticos variáves e os CCMs ocorrem em ambientes sinóticos mais homogêneas.

Calculou-se a correlação entre duração e área, para os SCMs alongados o coeficiente de correlação foi de 0.75 , portanto pode-se dizer que quanto maior a duração do sistema maior é a área que ele vai atingir, já para os CCMs não podemos afirmar isto, pois o coeficiente de correlação foi baixo, 0,57. A maior duração dos SCMs está associada a um ambiente baroclínico e a menor duração dos CCMs está associada a ambientes barotrópicos (ANABOR; FERREIRA, 2013).

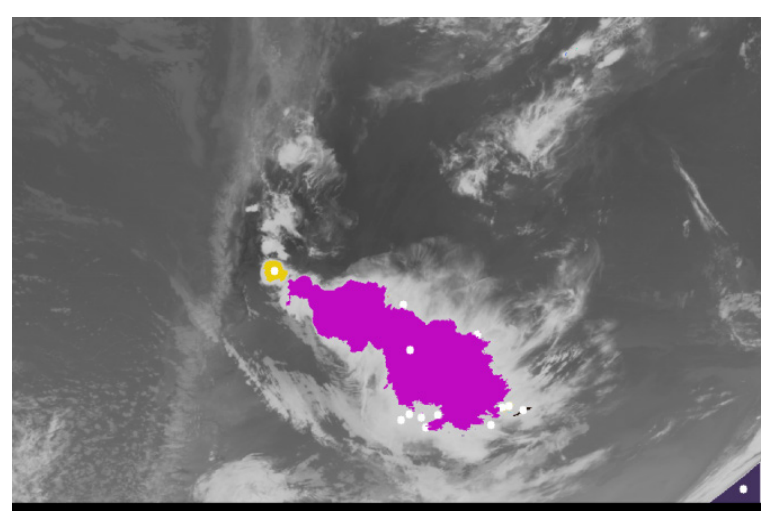

Figura 01- Imagem do satélite GOES 12, do dia 10/01/06, as 02:39 UTC. Mostra um SCM alongado no momento de sua área máxima

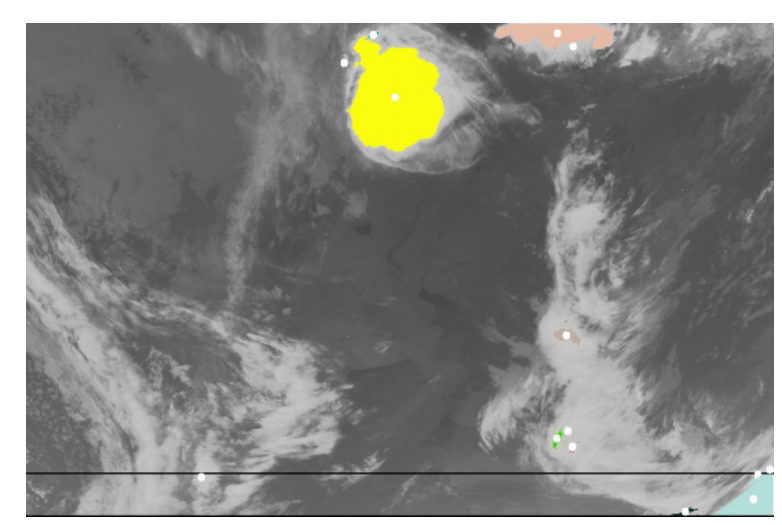

Figura 02 - Imagem do satélite GOES 12, do dia 15/12/06, as 08:45 UTC. Mostra um CCM no momento de sua área máxima

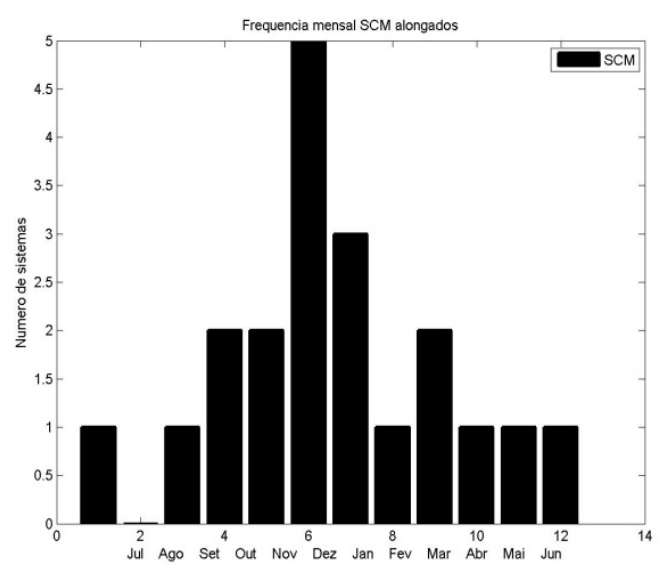

Figura 03 - Frequência mensal dos SCM alongados

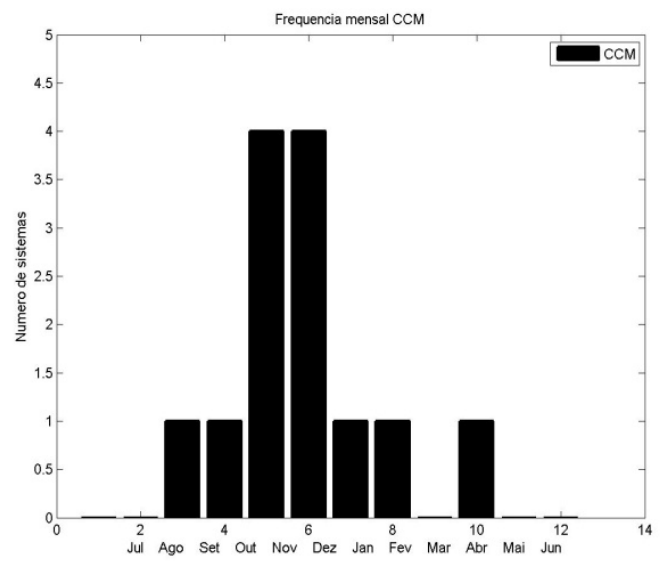

Figura 04 - Frequência mensal dos CCM 


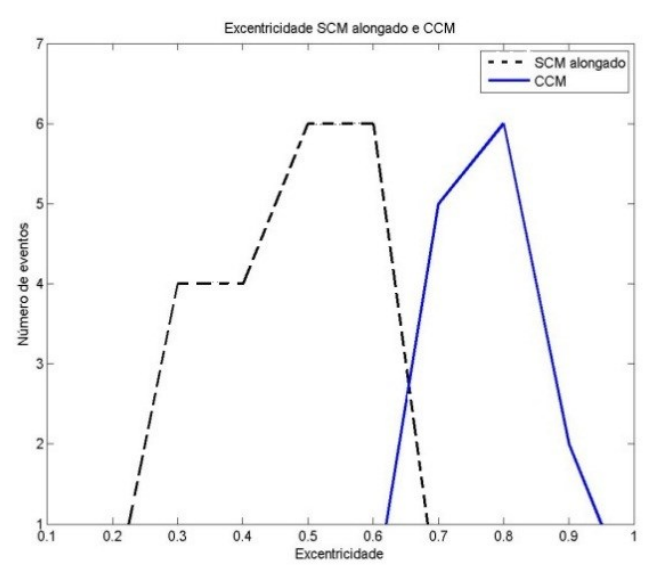

Figura 05 - Áreas máximas dos SCM alongados

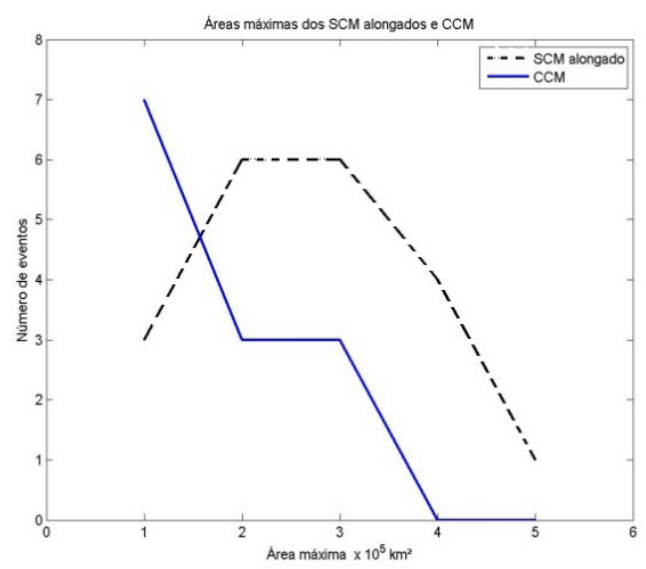

Figura 06 - Excentricidade no momento de área máxima dos SCM e CCM

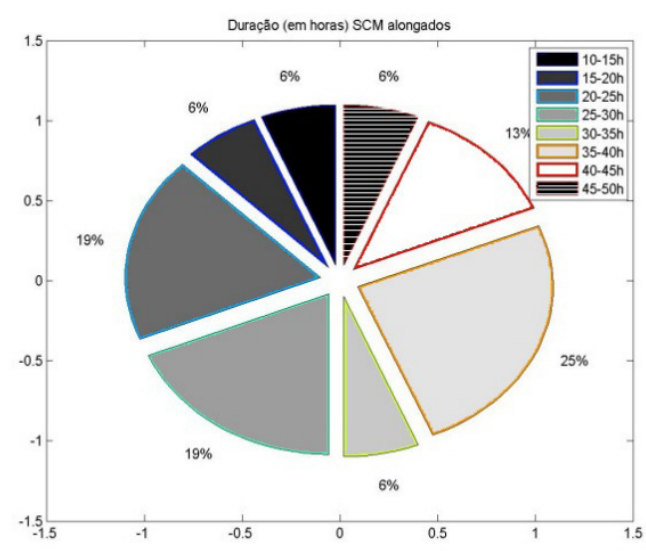

Figura 07 - Duração média em horas dos SCMs alongados

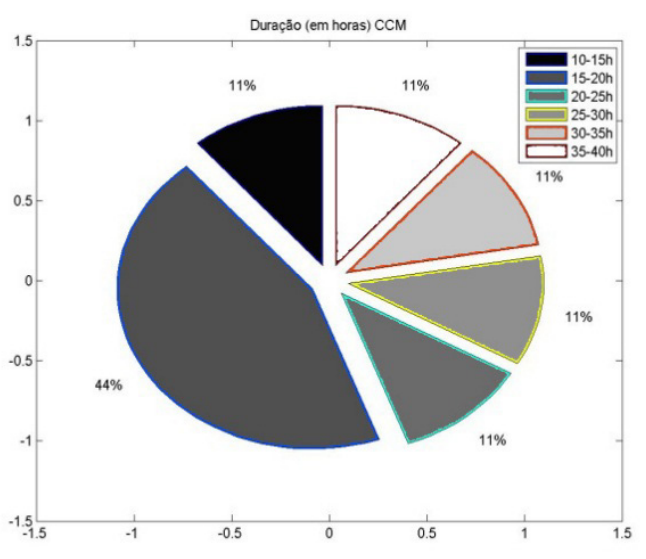

Figura 08 - Duração média em horas dos CCMs

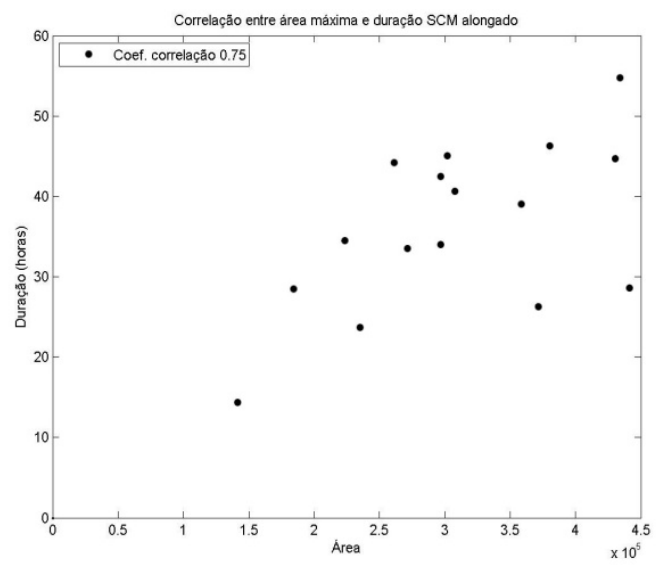

Figura 9 - Coeficiente de correlação entre área máxima e duração média dos SCMs alongados

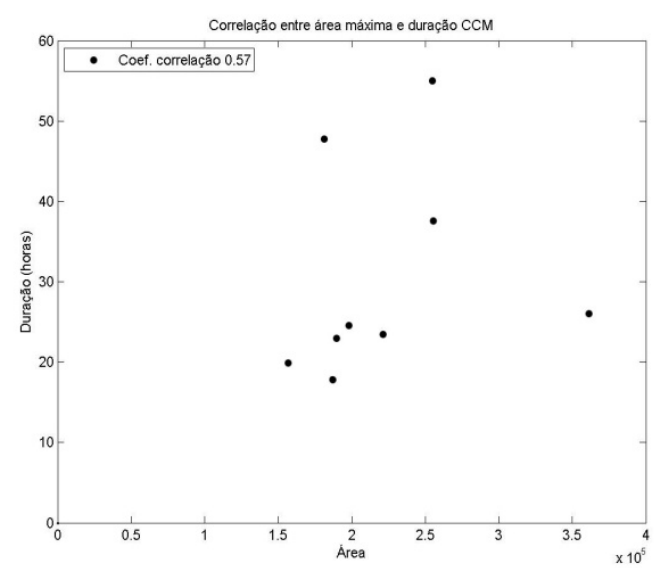

Figura 10 - Coeficiente de correlação e entre área máxima e duração média dos CCMs 


\section{Conclusão}

Pode-se concluir de um modo geral, que tanto SCMs alongados quanto CCMs ocorrem mais frequentemente nos meses quentes, entre outubro e março, e que os sistemas sobre a América do Sul são de longa duração, onde os SCMs alongados tiveram duração média de 44 horas e os CCMs de 26 horas.

Para os SCMs alongados há significativa correlação positiva entre duração e área, quanto maior a duração maior a área máxima atingida pelo sistema. Os SCMs alongados atingem áreas máximas maiores e tem duração maior que osCCMs. Também a curtose da distribuição da excentricidade dos sistemas alongados denota uma maior variância nesta medida, bem como uma maior variabilidade de condições atmosféricas que conduzem a ocorrência destes sistemas. Já os CCMs aparentemente ocorrem em situações atmosféricas com características mais homogêneas. Uma climatologia com período mais longo está sendo elaborada e permitirá inferir mais detalhes sobre o comportamento e ciclo de vida dos SCMs lineares e circulares.

\section{Referências}

Anabor, V., 2004: Descriptive analyses of meso- $\alpha$ convective systems by GOES-8 satellite images. M.S. thesis, Departament of Remote Sensing, Universidade Federal do Rio Grande do Sul, 78 pp.

Anabor, V., 2008: Sistemas Convectivos de Mesoescala no Sul da América do Sul: propagação em série contra o escoamento em baixos níveis. Tese de Doutorado, Departamento de Física, Universidade Federal de Santa Maria, $78 \mathrm{pp}$.

ANABOR, V., FERREIRA, V.: Características sinóticas médias dos Sistemas Convectivos de Mesoescala sobre a América do Sul no período de 2005 a 2006. V Simpósio Internacional de Climatologia, Florianópolis, SC,2013.

Anabor, V., D. J. Stensrud, and O. L. L de Moraes, 2008: Serial Upstream-propagating Mesoscale Convective System Events over Southeastern South America. Mon. Wea. Rev. Accepted 2007-11...In press.

Anderson, C. J., R. W. Arritt, 1998: Mesoscale convective complexes and persistent elongated convective systems over the United States during 1992 and 1993. Mon. Wea. Rev., 126, 578-599.

Durkee, J. D., Mote, T. L., 2010: A climatology of warmseason mesoscale convective

complexes in subtropical South America. International Journal of Climatology, 30, 418-431.
Maddox, R. A., 1980: Mesoscale convective complexes. Bull. Amer.Meteor. Soc., 61, 1374- 1387.

Maddox, R. A., 1983: Large-scale meteorological conditions associated with midlatitude, mesoscale convective complexes. Mon. Wea. Rev., 111, 126-140.

Velasco, I. Y., J. M. Fritsch, 1987: Mesoscale convective complexes in the Americas. J. Geophys. Res., 92, 95919613. 